\title{
Review Article \\ Facilitating Effects of Nanoparticles/Materials on Sensitive Immune-Related Lung Disorders
}

\author{
Ken-ichiro Inoue ${ }^{1}$ and Hirohisa Takano ${ }^{2}$ \\ ${ }^{1}$ Department of Public Health and Molecular Toxicology, School of Pharmacy Sciences, Kitasato University, 5-9-1 Shirokane, \\ Minato-ku, Tokyo 108-8641, Japan \\ ${ }^{2}$ Environmental Health Sciences Division, National Institute for Environmental Studies, Tsukuba, Ibaraki 305-8506, Japan
}

Correspondence should be addressed to Ken-ichiro Inoue, inouek@pharm.kitasato-u.ac.jp

Received 8 June 2010; Accepted 5 July 2010

Academic Editor: Hugh D. Smyth

Copyright ( $) 2011$ K.-i. Inoue and H. Takano. This is an open access article distributed under the Creative Commons Attribution License, which permits unrestricted use, distribution, and reproduction in any medium, provided the original work is properly cited.

\begin{abstract}
Although the adverse health effects of nanoparticles/materials have been proposed and are being clarified, their facilitating effects on preexisting pathological conditions have not been fully examined. In this paper, we provide insights into the immunotoxicity of nanoparticles/materials as an aggravating factor in hypersusceptible subjects, especially those with immune-related respiratory disorders using our in vivo experimental model. We first exhibit the effects of nanoparticles/materials on lung inflammation induced by bacterial endotoxin (lipopolysaccharide: LPS) in vivo as a disease model in innate immunity, and demonstrated that nanoparticles instilled through both an intratracheal tube and an inhalation system can exacerbate the lung inflammation. Secondly, we introduce the effects of nanoparticles/materials on allergic asthma in vivo as a disease model in adaptive immunity, and showed that repetitive pulmonary exposure to nanoparticles has aggravating effects on allergic airway inflammation, including adjuvant effects on Th2-milieu. Taken together, nanoparticle exposure may synergistically facilitate pathological inflammatory conditions in the lung via both innate and adaptive immunological abnormalities.
\end{abstract}

\section{Introduction}

Epidemiological studies have demonstrated a correlation between exposure to air pollutant particles at the concentrations currently found in major metropolitan areas and mortality and morbidity [1]. The concentration of particulate matter $(\mathrm{PM})$ with a mass median aerodynamic diameter (a density-dependent unit of measure used to describe the diameter of the particle $) \leqq 2.5 \mu \mathrm{m}\left(\mathrm{PM}_{2.5}\right)$ is more closely associated with both acute and chronic respiratory effects and subsequent mortality than larger particles of $\leqq 10 \mu \mathrm{m}\left(\mathrm{PM}_{10}\right)$ [2]. In addition, one intriguing aspect of the epidemiologic data is that health effects of $\mathrm{PM}_{2.5}$ are primarily seen in subjects with predisposing factors, including pneumonia, asthma, chronic obstructive pulmonary disease, compromised immune systems, atherosclerosis, age over 65 years old, and maybe depressive states [3-6]. Partially consistent with the epidemiological studies, we and others have experimentally demonstrated that diesel exhaust particles (DEP), major contributors to environmental $\mathrm{PM}_{2.5}$ in urban areas, exhibit respiratory toxicity with or without predisposing pathologies including allergic asthma, pulmonary emphysema, and acute renal failure in vivo [7-15].

To date, nanoparticles, particles less than $0.1 \mu \mathrm{m}$ in mass median aerodynamic diameter, have been shown to be increasing in ambient air [16]. Recent measurements indicate that nanoparticle numbers in ambient air range from $2 \times 10^{4} / \mathrm{cm}^{3}$ to $2 \times 10^{5} / \mathrm{cm}^{3}$, with mass concentrations of more than $50 \mu \mathrm{g} / \mathrm{m}^{3}$ near major highways $[17,18]$. Also, nanoparticles have been implicated in cardiopulmonary system effects [19]. Furthermore, compared to larger particles, nanoparticles have a higher deposition rate in the peripheral lung, can cross the pulmonary epithelium, reach the interstitium [20], and may thus be systemically distributed in the bloodstream [21]. Nanoparticles have an enhanced capacity to produce reactive oxygen species, and consequently have a widespread toxicity [22-24]. 
Further, nanotechnology is now advancing at such an incredible pace that it is has created an alternative industrial revolution over the past few years [25]. Consistent with this, the use of engineered nanomaterials has been rapidly increasing in commercial applications. As these materials have become more widespread, many questions have arisen regarding the adverse effects they may have on the environment as alternative inhalable toxicants. Due to their sizes, characteristics, and/or existing pattern, nanoparticles/materials have been implicated in cardiopulmonary system effects [19]. Compared to larger particles, nanoparticles have a higher deposition rate in the peripheral lung, can cross the pulmonary epithelium reach the interstitium [20], and, furthermore, may be systemically distributed in the bloodstream [21]. Furthermore, nanoparticles have an enhanced capacity to produce reactive oxygen species, and, consequently, have a widespread toxicity [22-24]. Nanoparticle exposure also reportedly influences cardiopulmonary systems in the presence or absence of predisposing diseases in human studies $[26,27]$. However, biological evidence concerning the promoting effects of nanoparticles/materials on predisposing subjects has been less studied. Besides their toxic effects on health, therefore, it should be experimentally ascertained whether they also aggravate preexisting pathological conditions, and their underlying mechanisms should be resolved. In this paper, therefore, we will discuss the impact of nanoparticles/materials as immunological enhancers.

\section{Effects of Nanoparticles on Acute Lung Inflammation Induced by Bacterial Endotoxin}

A glycolipid of gram-negative bacteria, known as endotoxin or lipopolysaccharide (LPS), stimulates host cells via innate immunity [28]. In animal models, intratracheal administration of LPS causes lung cytokine expression, neutrophil recruitment, and lung injury [29]. LPS is found in bronchoalveolar lavage (BAL) fluid of patients with pneumonia [30] and acute respiratory distress syndrome [31], which sometimes results in a fatal outcome. In addition, LPS is a significant constituent of many air pollutant particles and has accordingly been implicated in the adverse effects of PM [32]. In accordance with the close links among LPS, lung inflammation (injury), and PM, we have previously shown that intratracheal administration of DEPs and their components facilitates lung inflammation induced by LPS $[13,33]$ and subsequent systemic inflammation with coagulatory impairment [14].

We next examined the effects of pulmonary exposure to nanoparticles on lung inflammation related to LPS in mice. Vehicle, two sizes (14 and $56 \mathrm{~nm}$ ) of carbon black nanoparticles, LPS, or LPS + nanoparticles was administered intratracheally, and parameters of lung inflammation and coagulation were evaluated. Nanoparticles alone induced slight lung inflammation and significant pulmonary edema as compared with the vehicle. Fourteen-nm nanoparticles intensively aggravated LPS-elicited lung inflamma- tion and pulmonary edema, which was concomitant with the enhanced lung expression of interleukin (IL)- $1 \beta$, macrophage inflammatory protein (MIP)- $1 \alpha$, macrophage chemoattractant protein (MCP)-1, MIP-2, and keratinocyte chemoattractant $(\mathrm{KC})$ in overall trend, whereas $56-\mathrm{nm}$ nanoparticles did not show apparent effects. Immunoreactivity for 8-hydroxyguanosine ( $\mathrm{OHdG})$, a proper marker for oxidative stress, was more intense in the lung from the LPS + 14-nm nanoparticle group than that from the LPS group. The circulatory fibrinogen level was higher in the LPS + 14-nm nanoparticle group than that in the LPS group. Taken together, nanoparticles can aggravate lung inflammation related to bacterial endotoxin, which is more prominent with smaller particles. The enhancing effect may be mediated, at least partly, via the increased local expression of proinflammatory cytokines and via the oxidative stress. Furthermore, nanoparticles can promote coagulatory disturbance accompanied by lung inflammation [34].

Furthermore, we examined the adverse effects of nanomaterials on this pathological model. In brief, ICR male mice were divided into 8 experimental groups that intratracheally received vehicle, three sizes $(15,50,100 \mathrm{~nm})$ of $\mathrm{TiO}_{2}$ nanomaterials, LPS, or LPS plus nanomaterials. Twenty four hours after the treatment, both nanomaterials exacerbated the lung inflammation and edema elicited by LPS, with an overall trend of amplified lung expressions of cytokines such as IL-1 $\beta$, MCP-1, and KC. LPS plus nanomaterials, especially with size less than $50 \mathrm{~nm}$, elevated circulatory levels of fibrinogen, IL- $1 \beta$, MCP-1, KC, and von Willebrand factor as compared with LPS alone. The enhancement tended overall to be greater with the smaller nanomaterials than that with the larger ones. cDNA microarray analyses revealed that gene expression pattern was not different between the LPS group and the LPS + nanomaterial groups. These results suggest that nanomaterials exacerbate lung inflammation related to LPS with systemic inflammation and coagulatory impairment, and the exacerbation is more prominent with smaller nanomaterials than that with larger ones ([35] and unpublished data). Additionally, we demonstrated that latex nanoparticles [36] and carbon nanotubes [37] have similar adverse effects on the lung pathophysiology.

Our next study was conducted to determine whether inhaled exposure to diesel engine-derived nanoparticles also exacerbates the model. ICR mice were exposed for 5 hours to clean air or diesel engine-derived nanoparticles at a concentration of 15,36 , or $169 \mu \mathrm{g} / \mathrm{m}^{3}$ after intratracheal challenge with LPS or vehicle, and were sacrificed for evaluation 24 hours after the intratracheal challenge. Exposure to nanoparticles alone did not elicit lung inflammation. Nanoparticle inhalation exaggerated LPS-elicited inflammatory cell recruitment in the BAL fluid and lung parenchyma as compared with clean air inhalation in a concentrationdependent manner. Lung homogenates derived from the LPS + nanoparticle groups tended to have an increased tumor necrosis factor- $\alpha$ level and chemotaxis activity for polymorphonuclear leukocytes as compared with those from the LPS group or the corresponding nanoparticle groups. Nanoparticle inhalation did not significantly increase the lung expression of proinflammatory cytokines or influence 


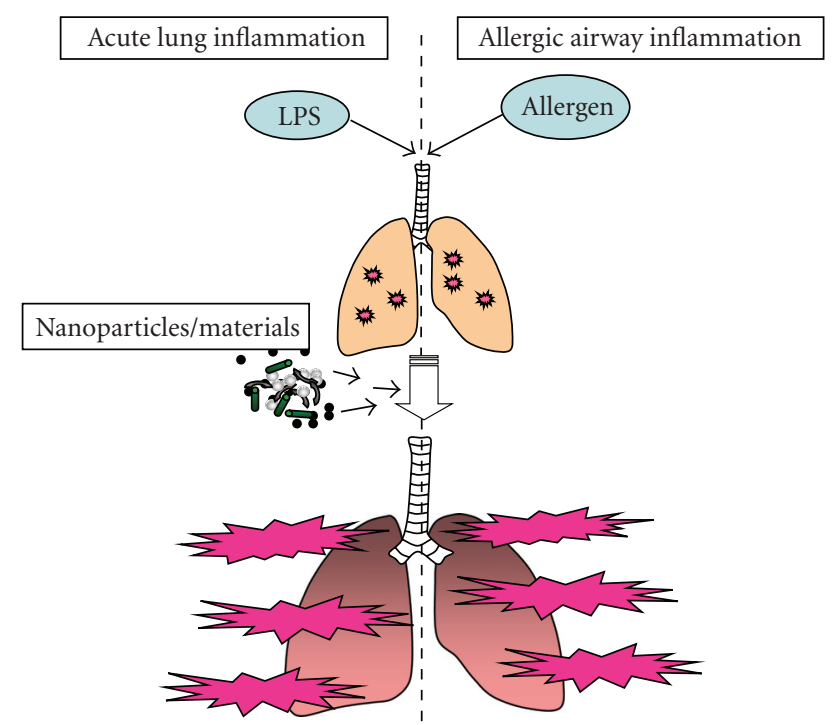

Figure 1: Proposal schema for enhancement of nanoparticles/materials on sensitive immune-related lung disorders.

systemic inflammation. Isolated alveolar macrophages from nanoparticle-exposed mice showed a greater production of IL-1 $\beta$ and KC stimulated with ex vivo LPS challenge than those from clean air-exposed mice although the differences did not reach significance. These results suggest that acute exposure to diesel-nanoparticles exacerbates lung inflammation induced by LPS [38]. In sum, nanoparticle/material exposure exacerbates acute lung inflammation related to bacterial endotoxin (Figure 1).

\section{Effects of Nanoparticles on Allergic Airway Inflammation}

Bronchial asthma has been recognized as chronic airway inflammation with hyperresponsiveness that is characterized by an increase in the number of activated lymphocytes and eosinophils [39]. A number of studies have shown that various particles including carbon black can enhance allergic sensitization [40-42], which is referred to as "adjuvant effect" as well. Carbon black has been demonstrated to enhance the proliferation of antibody-forming cells and both IgE and IgG levels [43, 44]. Ultrafine particles (PM and carbon black) reportedly exaggerate allergic airway inflammation in vivo $[45,46]$. However, all studies have failed to pay attention to the size of particles. Therefore, no research has addressed the size effects of particles or nanoparticles on airway biology in the presence or absence of allergen in vivo. Given the hypothesis, we investigated the effects of carbon black nanoparticles with a diameter of $14 \mathrm{~nm}$ or $56 \mathrm{~nm}$ on allergen-related airway inflammation. ICR mice were divided into six experimental groups. Vehicle, two sizes of carbon nanoparticles, ovalbumin (OVA) and OVA + nanoparticles, were administered intratracheally. The cellular profile of BAL fluid, lung histology, expression of cytokines, chemokines, 8-OHdG, and immunoglobulin production were studied.
Nanoparticles with a diameter of $14 \mathrm{~nm}$ or $56 \mathrm{~nm}$ aggravated antigen-related airway inflammation characterized by the infiltration of eosinophils, neutrophils, and mononuclear cells, and by an increase in the number of goblet cells in the bronchial epithelium. Nanoparticles with antigen increased protein levels of IL-5, IL-6, IL-13, eotaxin, MCP-1, and regulated upon activation and normal T-cells expressed and secreted (RANTES) in the lung as compared with antigen alone. The formation of 8-OHdG was moderately induced by nanoparticles or allergen alone, and was markedly enhanced by allergen plus nanoparticles as compared with nanoparticles or allergen alone. The aggravation was more prominent with $14 \mathrm{~nm}$ nanoparticles than that with $56-\mathrm{nm}$ particles in terms of the overall trend. Particles with a diameter of $14 \mathrm{~nm}$ exhibited an adjuvant activity for total $\operatorname{IgE}$ and antigen-specific IgG and IgE. Nanoparticles can aggravate allergen-related airway inflammation and immunoglobulin production, which become more prominent with smaller particles. The enhancement may be mediated, at least partly, by the increased local expression of IL- 5 and eotaxin, and also by the modulated expression of IL-13, RANTES, MCP-1, and IL-6 [47]. Consistent with our study, de Haar and colleagues have previously shown that nanoparticles (14 and $29 \mathrm{~nm}$ ) potently facilitate allergic airway inflammation as compared with fine particles (250 and $260 \mathrm{~nm}$ ) [48].

In ongoing reports, nanoparticles alone or OVA alone moderately enhanced cholinergic airway reactivity, as assessed by total respiratory system resistance $(R)$ and Newtonian resistance $\left(R_{n}\right)$. All the parameters of lung responsiveness, such as $R$, compliance, elastance, $R_{n}$, tissue damping, and tissue elastance, were worse in the OVA + nanoparticle groups than those in the vehicle group, the corresponding nanoparticle groups, or the OVA group. The lung mRNA level for Muc5ac was significantly higher in the OVA group than that in the vehicle group, and further increased in the OVA + nanoparticle groups than that in the OVA or nanoparticle groups. These data suggest that carbon nanoparticles can enhance lung hyperresponsiveness, especially in the presence of allergen. The effects may be mediated, at least partly, through the enhanced lung expression of Muc5ac [49].

Furthermore, we recently demonstrated that (singlewalled and multiwalled) carbon nanotubes promote allergic airway inflammation in mice, which may be partly through enhanced oxidative stress in the airway and the inappropriate activation of antigen-presenting cells including dendritic cells (in vitro) $[50,51]$. In addition, other groups have reported the similar impacts of nanomaterials (carbon nanotubes, $\mathrm{TiO}_{2}$, and on gold) us as on animal allergic asthma models [48, 52-54]. Moreover, as for cellular contribution, we and others have claimed that antigen-presenting cells such as dendritic cells are important target cell populations for the adjuvant activity of nanoparticles/materials [5557]. Taken together, nanoparticle/material exposure can exacerbate allergic asthma (Figure 1).

\section{Considerations for Future Directions}

4.1. Risk Factors Regarding Nanoparticles. One important point to be taken into consideration in these studies is the 
surface characteristics and numbers of nanoparticles used. Our results indicate that nanoparticles, particularly smaller ones (14 $\mathrm{nm}$ in diameter), can aggravate lung inflammation related to LPS and allergic airway inflammation when the weights of particles are equal. On the other hand, the surface area of the 14-nm nanoparticles was 6.7 fold larger than that of $56-\mathrm{nm}$ nanoparticles ( $300 \mathrm{~m}^{2} / \mathrm{g}$ versus $45 \mathrm{~m}^{2} / \mathrm{g}$, resp.). The surface area of particles exposed to is reportedly correlated with lung inflammation [37]. Alternatively, our studies have demonstrated not only the size effects of nanoparticles on lung inflammation, but also the effects of their surface area and/or their numbers on the inflammation.

Unfortunately, we could not examine the effects of nanoparticles with the same particle number in these studies. The number of smaller nanoparticles is greater than that of larger nanoparticles when the particles comprise the same weight.

\subsection{Possibility of Migration and Influence of Nanoparticles} Exposed to the Airway into Systemic Circulation. It also remains to be argued whether nano-level particles/materials delivered through the airway enter the systemic circulation and cause serve adverse effects such as systemic inflammation and thrombus formation. Nanoparticles are reportedly able to penetrate deeply into the respiratory tract and can even pass the lung to reach systemic circulation $[58,59]$. Nemmar et al. have previously demonstrated that nanoparticles can migrate into the circulation [59]. In our study, the LPS + nanoparticle groups, specifically the LPS + 14-nm nanoparticle group, showed significantly higher fibrinogen levels when compared to the LPS group. Additionally, although not significant, the enhanced activity of vWF induced by LPS was further increased by its combination with 14-nm nanoparticles [34]. These findings suggest that smaller nanoparticles can facilitate coagulatory disturbance accompanied by lung inflammation. The enhancing effects of 14-nm nanoparticles on LPS-elicited pulmonary edema further support this concept. Interestingly, exposure to nanoparticles alone did not induce significant fibrinogen production/release nor did it activate vWF. It might be hypothesized that endothelialepithelial damage induced by LPS and subsequent infiltrating effector leukocytes allow a large amount of smaller nanoparticles to pass easily into the circulation, resulting in synergistic effects on hemostasis including coagulatory disturbance. On the other hand, exposure to environmental particles reportedly generates local and systemic oxidative stress, which, in turn, induces/enhances inflammation and blood coagulation [58]. Further, Nemmar and colleagues have demonstrated that nanoparticles instilled intratracheally rapidly diffuse from the lung into the systemic circulation in vivo [59]. Therefore, it is also possible that intratracheally instilled nanoparticles enter the circulation by themselves and contribute to a high susceptibility to LPSelicited systemic inflammation and coagulatory disturbance. Future studies are needed to confirm the penetration and address the above-mentioned hypothesis.

4.3. Model's Relevance to the Actual Situation. In reality, we inhale suspended particulate matters in ambient air, but do not intratracheally receive them in aliquot. Nevertheless, the impacts of inhalation exposure to these particles/materials, the more realistic exposure, on this lung inflammation model had less been conducted by us and others. In our previous study, nanoparticle-rich diesel exhaust inhalation exaggerated lung inflammation induced by LPS [38]. Nonetheless, we have not completed/examined the effects of the inhalation on other disease models. In future, therefore, more realistic research considering the effects of the mode of nanoparticles/materials administration (instillation versus aerosolization, droplets versus powder, etc.) on the in vivo response would be very valuable to toxicologists, environmental scientists, and immunologists.

\section{Acknowledgments}

Special thanks to Professor Takamichi Ichinose, Professor Seishiro Hirano, Dr. Rie Yanagisawa, and Dr. Eiko Koike for greatly contributing to the work in the paper. The authors are supported by NIES Grants.

\section{References}

[1] J. M. Samet, F. Dominici, F. C. Curriero, I. Coursac, and S. L. Zeger, "Fine particulate air pollution and mortality in 20 U.S. cities, 1987-1994," New England Journal of Medicine, vol. 343, no. 24, pp. 1742-1749, 2000.

[2] A. Peters, H. E. Wichmann, T. Tuch, J. Heinrich, and J. Heyder, "Respiratory effects are associated with the number of ultrafine particles," American Journal of Respiratory and Critical Care Medicine, vol. 155, no. 4, pp. 1376-1383, 1997.

[3] M. Szyszkowicz, "Air pollution and emergency department visits for depression in Edmonton, Canada," International Journal of Occupational Medicine and Environmental Health, vol. 20, no. 3, pp. 241-245, 2007.

[4] N. Künzli, P.-O. Bridevaux, L.-J. S. Liu et al., "Traffic-related air pollution correlates with adult-onset asthma among neversmokers," Thorax, vol. 64, no. 8, pp. 664-670, 2009.

[5] B. Neupane, M. Jerrett, R. T. Burnett, T. Marrie, A. Arain, and M. Loeb, "Long-term exposure to ambient air pollution and risk of hospitalization with community-acquired pneumonia in older adults," American Journal of Respiratory and Critical Care Medicine, vol. 181, no. 1, pp. 47-53, 2010.

[6] D. W. Dockery, C. A. Pope III, X. Xu et al., "An association between air pollution and mortality in six U.S. cities," New England Journal of Medicine, vol. 329, no. 24, pp. 1753-1759, 1993.

[7] A. Nemmar, S. Al-Salam, S. Zia, J. Yasin, I. Al Husseni, and B. H. Ali, "Diesel exhaust particles in the lung aggravate experimental acute renal failure," Toxicological Sciences, vol. 113, no. 1, pp. 267-277, 2009.

[8] F. D. T. Q. S. Lopes, T. S. Pinto, F. M. Arantes-Costa et al., "Exposure to ambient levels of particles emitted by traffic worsens emphysema in mice," Environmental Research, vol. 109, no. 5, pp. 544-551, 2009.

[9] K.-I. Inoue, H. Takano, R. Yanagisawa, T. Ichinose, A. Shimada, and T. Yoshikawa, "Pulmonary exposure to diesel exhaust particles induces airway inflammation and cytokine expression in NC/Nga mice," Archives of Toxicology, vol. 79, no. 10, pp. 595-599, 2005. 
[10] T. Ichinose, A. Furuyama, and M. Sagai, "Biological effects of diesel exhaust particles (DEP). II. Acute toxicity of DEP introduced into lung by intratracheal instillation," Toxicology, vol. 99, no. 3, pp. 153-167, 1995.

[11] T. Ichinose, Y. Yajima, M. Nagashima, S. Takenoshita, Y. Nagamachi, and M. Sagai, "Lung carcinogenesis and formation of 8-hydroxy-deoxyguanosine in mice by diesel exhaust particles," Carcinogenesis, vol. 18, no. 1, pp. 185-192, 1997.

[12] H. Takano, T. Yoshikawa, T. Ichinose, Y. Miyabara, K. Imaoka, and M. Sagai, "Diesel exhaust particles enhance antigeninduced airway inflammation and local cytokine expression in mice," American Journal of Respiratory and Critical Care Medicine, vol. 156, no. 1, pp. 36-42, 1997.

[13] H. Takano, R. Yanagisawa, T. Ichinose et al., "Diesel exhaust particles enhance lung injury related to bacterial endotoxin through expression of proinflammatory cytokines, chemokines, and intercellular adhesion molecule-1," American Journal of Respiratory and Critical Care Medicine, vol. 165, no. 9, pp. 1329-1335, 2002.

[14] K.-I. Inoue, H. Takano, M. Sakurai et al., "Pulmonary exposure to diesel exhaust particles enhances coagulatory disturbance with endothelial damage and systemic inflammation related to lung inflammation," Experimental Biology and Medicine, vol. 231, no. 10, pp. 1626-1632, 2006.

[15] K.-I. Inoue, E. Koike, H. Takano, R. Yanagisawa, T. Ichinose, and T. Yoshikawa, "Effects of diesel exhaust particles on antigen-presenting cells and antigen-specific Th immunity in mice," Experimental Biology and Medicine, vol. 234, no. 2, pp. 200-209, 2009.

[16] J. Cyrys, M. Stölzel, J. Heinrich et al., "Elemental composition and sources of fine and ultrafine ambient particles in Erfurt, Germany," Science of the Total Environment, vol. 305, no. 1-3, pp. 143-156, 2003.

[17] K. L. Timonen, G. Hoek, J. Heinrich et al., "Daily variation in fine and ultrafine particulate air pollution and urinary concentrations of lung Clara cell protein CC16," Occupational and Environmental Medicine, vol. 61, no. 11, pp. 908-914, 2004.

[18] Y. Zhu, W. C. Hinds, S. Kim, and C. Sioutas, "Concentration and size distribution of ultrafine particles near a major highway," Journal of the Air and Waste Management Association, vol. 52, no. 9, pp. 1032-1042, 2002.

[19] M. J. Utell and M. W. Frampton, "Acute health effects of ambient air pollution: the ultrafine particle hypothesis," Journal of Aerosol Medicine, vol. 13, no. 4, pp. 355-359, 2000.

[20] G. Oberdörster, "Pulmonary effects of inhaled ultrafine particles," International Archives of Occupational and Environmental Health, vol. 74, no. 1, pp. 1-8, 2001.

[21] A. Seaton, W. MacNee, K. Donaldson, and D. Godden, "Particulate air pollution and acute health effects," Lancet, vol. 345, no. 8943, pp. 176-178, 1995.

[22] D. M. Brown, M. R. Wilson, W. MacNee, V. Stone, and K. Donaldson, "Size-dependent proinflammatory effects of ultrafine polystyrene particles: a role for surface area and oxidative stress in the enhanced activity of ultrafines," Toxicology and Applied Pharmacology, vol. 175, no. 3, pp. 191-199, 2001.

[23] C. A. J. Dick, D. M. Brown, K. Donaldson, and V. Stone, "The role of free radicals in the toxic and inflammatory effects of four different ultrafine particle types," Inhalation Toxicology, vol. 15, no. 1, pp. 39-52, 2003.

[24] N. Li, C. Sioutas, A. Cho et al., "Ultrafine particulate pollutants induce oxidative stress and mitochondrial damage," Environmental Health Perspectives, vol. 111, no. 4, pp. 455-460, 2003.
[25] R. F. Service, "Nanotechnology grows up," Science, vol. 304, no. 5678, pp. 1732-1734, 2004.

[26] H. Gong Jr., W. S. Linn, K. W. Clark et al., "Exposures of healthy and asthmatic volunteers to concentrated ambient ultrafine particles in Los Angeles," Inhalation Toxicology, vol. 20, no. 6, pp. 533-545, 2008.

[27] M. W. Frampton, J. C. Stewart, G. Oberdörster et al., "Inhalation of ultrafine particles alters blood leukocyte expression of adhesion molecules in humans," Environmental Health Perspectives, vol. 114, no. 1, pp. 51-58, 2006.

[28] M. P. Vincenti, T. A. Burrell, and S. M. Taffet, "Regulation of NF- $\kappa$ B activity in murine macrophages: effect of bacterial lipopolysaccharide and phorbol ester," Journal of Cellular Physiology, vol. 150, no. 1, pp. 204-213, 1992.

[29] T. R. Ulich, L. R. Watson, S. Yin et al., "The intratracheal administration of endotoxin and cytokines: I. Characterization of LPS-induced IL-1 and TNF mRNA expression and the LPS-, IL-1-, and TNF-induced inflammatory infiltrate," American Journal of Pathology, vol. 138, no. 6, pp. 1485-1496, 1991.

[30] P. G. Flanagan, S. K. Jackson, and G. Findlay, "Diagnosis of gram negative, ventilator associated pneumonia by assaying endotoxin in bronchial lavage fluid," Journal of Clinical Pathology, vol. 54, no. 2, pp. 107-110, 2001.

[31] T. R. Martin, G. D. Rubenfeld, J. T. Ruzinski et al., "Relationship between soluble CD14, lipopolysaccharide binding protein, and the alveolar inflammatory response in patients with acute respiratory distress syndrome," American Journal of Respiratory and Critical Care Medicine, vol. 155, no. 3, pp. 937-944, 1997.

[32] S. Becker, M. J. Fenton, and J. M. Soukup, "Involvement of microbial components and toll-like receptors 2 and 4 in cytokine responses to air pollution particles," American Journal of Respiratory Cell and Molecular Biology, vol. 27, no. 5, pp. 611-618, 2002.

[33] R. Yanagisawa, H. Takano, K. Inoue et al., "Enhancement of acute lung injury related to bacterial endotoxin by components of diesel exhaust particles," Thorax, vol. 58, no. 7, pp. 605-612, 2003.

[34] K.-I. Inoue, H. Takano, R. Yanagisawa et al., "Effects of airway exposure to nanoparticles on lung inflammation induced by bacterial endotoxin in mice," Environmental Health Perspectives, vol. 114, no. 9, pp. 1325-1330, 2006.

[35] K.-I. Inoue, H. Takano, M. Ohnuki et al., "Size effects of nanomaterials on lung inflammation and coagulatory disturbance," International Journal of Immunopathology and Pharmacology, vol. 21, no. 1, pp. 197-206, 2008.

[36] K.-I. Inoue, H. Takano, R. Yanagisawa, E. Koike, and A. Shimada, "Size effects of latex nanomaterials on lung inflammation in mice," Toxicology and Applied Pharmacology, vol. 234, no. 1, pp. 68-76, 2009.

[37] K.-I. Inoue, H. Takano, E. Koike et al., "Effects of pulmonary exposure to carbon nanotubes on lung and systemic inflammation with coagulatory disturbance induced by lipopolysaccharide in mice," Experimental Biology and Medicine, vol. 233, no. 12, pp. 1583-1590, 2008.

[38] K.-I. Inoue, H. Takano, R. Yanagisawa et al., "Effects of inhaled nanoparticles on acute lung injury induced by lipopolysaccharide in mice," Toxicology, vol. 238, no. 2-3, pp. 99-110, 2007.

[39] D. K. Agrawal and Z. Shao, "Pathogenesis of allergic airway inflammation," Current Allergy and Asthma Reports, vol. 10, no. 1, pp. 39-48, 2010. 
[40] K. Maejima, K. Tamura, Y. Taniguchi, S. Nagase, and H. Tanaka, "Comparison of the effects of various fine particles on IgE antibody production in mice inhaling Japanese cedar pollen allergens," Journal of Toxicology and Environmental Health, Part A, vol. 52, no. 3, pp. 231-248, 1997.

[41] A. L. Lambert, W. Dong, D. W. Winsett, M. K. Selgrade, and M. I. Gilmour, "Residual oil fly ash exposure enhances allergic sensitization to house dust mite," Toxicology and Applied Pharmacology, vol. 158, no. 3, pp. 269-277, 1999.

[42] A. L. Lambert, W. Dong, M. K. Selgrade, and M. I. Gilmour, "Enhanced allergic sensitization by residual oil fly ash particles is mediated by soluble metal constituents," Toxicology and Applied Pharmacology, vol. 165, no. 1, pp. 84-93, 2000.

[43] M. Løvik, A.-K. Høgseth, P. I. Gaarder, R. Hagemann, and I. Eide, "Diesel exhaust particles and carbon black have adjuvant activity on the local lymph node response and systemic IgE production to ovalbumin," Toxicology, vol. 121, no. 2, pp. 165178, 1997.

[44] M. Van Zijverden, A. Van der Pijl, M. Bol et al., "Diesel exhaust, carbon black, and silica particles display distinct Th1/Th2 modulating activity," Toxicology and Applied Pharmacology, vol. 168, no. 2, pp. 131-139, 2000.

[45] J. A. Last, R. Ward, L. Temple, K. E. Pinkerton, and N. J. Kenyon, "Ovalbumin-induced airway inflammation and fibrosis in mice also exposed to ultrafine particles," Inhalation Toxicology, vol. 16, no. 2, pp. 93-102, 2004.

[46] N. H. Al-Humadi, P. D. Siegel, D. M. Lewis et al., "The effect of diesel exhaust particles (DEP) and carbon black (CB) on thiol changes in pulmonary ovalbumin allergic sensitized brown norway rats," Experimental Lung Research, vol. 28, no. 5, pp. 333-349, 2002.

[47] K.-I. Inoue, H. Takano, R. Yanagisawa et al., "Effects of nano particles on antigen-related airway inflammation in mice," Respiratory Research, vol. 6, article 106, 2005.

[48] C. De Haar, I. Hassing, M. Bol, R. Bleumink, and R. Pieters, "Ultrafine but not fine particulate matter causes airway inflammation and allergic airway sensitization to coadministered antigen in mice," Clinical and Experimental Allergy, vol. 36, no. 11, pp. 1469-1479, 2006.

[49] K. Inoue, H. Takano, R. Yanagisawa et al., "Effects of nanoparticles on lung physiology in the presence or absence of antigen," International Journal of Immunopathology and Pharmacology, vol. 20, no. 4, pp. 737-744, 2007.

[50] K.-I. Inoue, E. Koike, R. Yanagisawa, S. Hirano, M. Nishikawa, and H. Takano, "Effects of multi-walled carbon nanotubes on a murine allergic airway inflammation model," Toxicology and Applied Pharmacology, vol. 237, no. 3, pp. 306-316, 2009.

[51] K. Inoue, R. Yanagisawa, E. Koike, M. Nishikawa, and H. Takano, "Repeated pulmonary exposure to single-walled carbon nanotubes exacerbates allergic inflammation of the airway: possible role of oxidative stress," Free Radical Biology and Medicine, vol. 48, no. 7, pp. 924-934, 2010.

[52] U. C. Nygaard, J. S. Hansen, M. Samuelsen, T. Alberg, C. D. Marioara, and M. Løvik, "Single-walled and multi-walled carbon nanotubes promote allergic immune responses in mice," Toxicological Sciences, vol. 109, no. 1, pp. 113-123, 2009.

[53] J. P. Ryman-Rasmussen, E. W. Tewksbury, O. R. Moss, M. F. Cesta, B. A. Wong, and J. C. Bonner, "Inhaled multiwalled carbon nanotubes potentiate airway fibrosis in murine allergic asthma," American Journal of Respiratory Cell and Molecular Biology, vol. 40, no. 3, pp. 349-358, 2009.

[54] S. Hussain, J. A. Vanoirbeek, K. Luyts et al., "Lung exposure to nanoparticles modulates an asthmatic response in a mouse model of asthma," European Respiratory Journal. In press.
[55] E. Koike, H. Takano, K.-I. Inoue, R. Yanagisawa, and T. Kobayashi, "Carbon black nanoparticles promote the maturation and function of mouse bone marrow-derived dendritic cells," Chemosphere, vol. 73, no. 3, pp. 371-376, 2008.

[56] E. Koike, H. Takano, K.-I. Inoue et al., "Pulmonary exposure to carbon black nanoparticles increases the number of antigenpresenting cells in murine lung," International Journal of Immunopathology and Pharmacology, vol. 21, no. 1, pp. 35-42, 2008.

[57] C. de Haar, M. Kool, I. Hassing, M. Bol, B. N. Lambrecht, and R. Pieters, "Lung dendritic cells are stimulated by ultrafine particles and play a key role in particle adjuvant activity," Journal of Allergy and Clinical Immunology, vol. 121, no. 5, pp. 1246-1254, 2008.

[58] W. MacNee and K. Donaldson, "How can ultrafine particles be responsible for increased mortality?" Monaldi Archives for Chest Disease, vol. 55, no. 2, pp. 135-139, 2000.

[59] A. Nemmar, H. Vanbilloen, M. F. Hoylaerts, P. H. M. Hoet, A. Verbruggen, and B. Nemery, "Passage of intratracheally instilled ultrafine particles from the lung into the systemic circulation in hamster," American Journal of Respiratory and Critical Care Medicine, vol. 164, no. 9, pp. 1665-1668, 2001. 

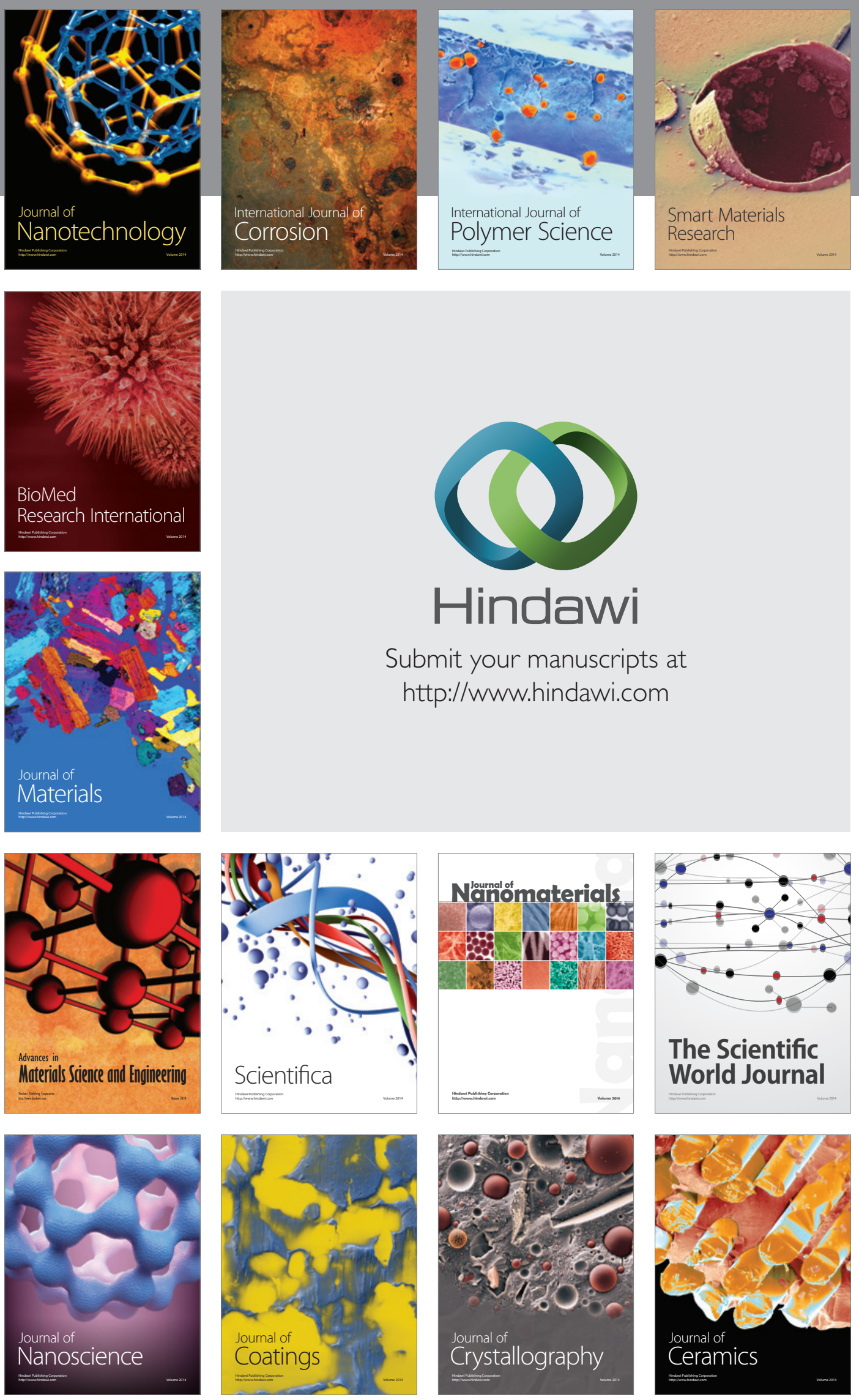

The Scientific World Journal

Submit your manuscripts at

http://www.hindawi.com

\section{World Journal}

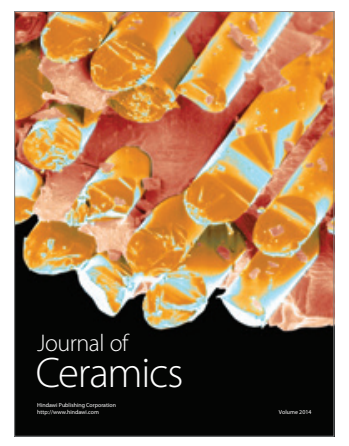

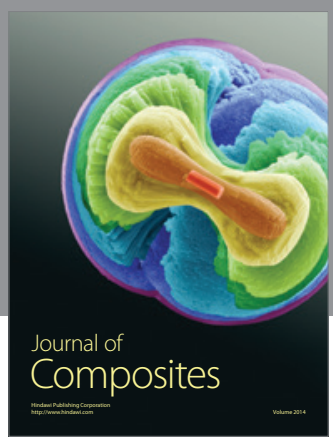
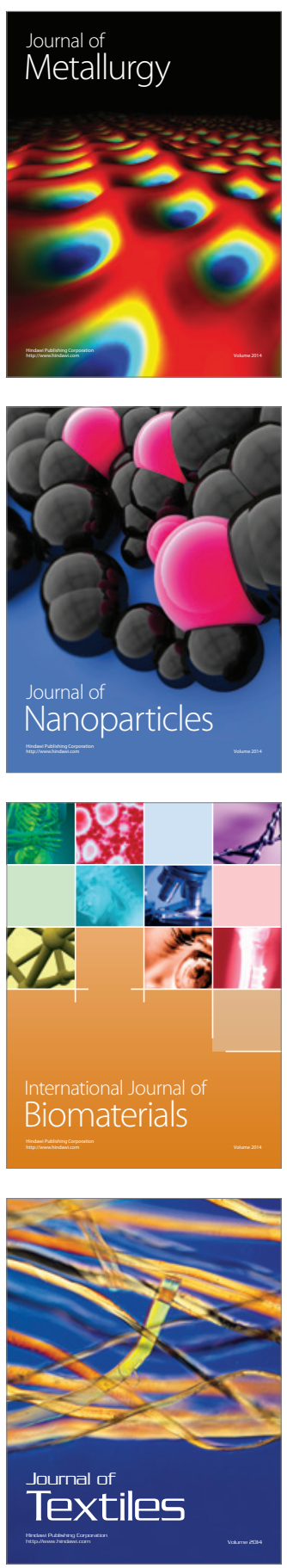